\title{
COMPOSIÇÃO FLORÍSTICA E ESTRATÉGIAS DE DISPERSÃO E REGENERAÇÃO DE GRUPOS FLORÍSTICOS EM FLORESTAS ESTACIONAIS DECIDUAIS NO RIO GRANDE DO SUL
}

\author{
Marcelo Callegari Scipioni ${ }^{1}$, Franklin Galvão ${ }^{2}$, Solon Jonas Longhi ${ }^{3}$ \\ ${ }^{1}$ Eng. Florestal, Dr., Curso de Engenharia Florestal, UFSC, Curitibanos, SC, Brasil - marcelo.scipioni@gmail.com \\ ${ }^{2}$ Eng. Florestal, Dr., Depto. de Ciências Florestais, UFPR, Curitiba, PR, Brasil - fgalvao@ufpr.br \\ ${ }^{3}$ Eng. Florestal, Dr., Depto. de Ciências Florestais, UFSM, Santa Maria, RS, Brasil - longhi.solon@ gmail.com
}

Recebido para publicação: 23/06/2012 - Aceito para publicação: 26/03/2013

\begin{abstract}
Resumo
As Florestas Estacional Decidual Aluvial e Submontana encontram-se ao longo de um gradiente topográfico com variações geomorfológicas e pedológicas. Para entender as variações das guildas ecológicas e do contingente geográfico das comunidades florestais em uma microbacia do rio Jacuí, no Rio Grande do Sul, foram alocadas 200 parcelas de $100 \mathrm{~m}^{2}$, distribuídas em 40 pontos amostrais, com 5 parcelas cada, desde as cabeceiras de drenagem até a planície de inundação do referido rio. Em cada unidade amostral foram medidos e identificados todos os indivíduos lenhosos com diâmetro à altura do peito de $\geq 5 \mathrm{~cm}$. A partir da amostra total, foi realizada uma análise de agrupamento de similaridade florística, resultando em três comunidades florestais distintas. Elas foram nomeadas de formações aluvial e submontana e área dominada por ambas as formações. Para a amostra total e dos grupos obtidos, foram determinadas as porcentagens de contribuição das espécies e indivíduos, por meio da classificação de guildas ecológicas de dispersão de diásporos e grupos ecológicos, além dos contingentes fitogeográficos. O contingente geográfico de composição das comunidades florestais foi dominado por espécies de ampla distribuição. Os padrões das guildas ecológicas entre os grupos foram distintos. Entretanto, todos os grupos apresentaram maior contribuição de espécies zoocóricas. Palavras-chave: Comunidades florestais; fitogeografia; similaridades florísticas; ecologia florestal.
\end{abstract}

\begin{abstract}
Floristic composition and dispersal and regeneration strategies of woody species in Deciduous Seasonal Forests. The connected fragments of submontane and alluvial deciduous forests are present along the topographic gradient with large pedological and geomorphological variations. In order to understand the variations of ecological guilds and geographical contingent of forest community in a watershed, 200 plots of $100 \mathrm{~m}^{2}$ divided into 40 sampling points with five plots each were distributed from the headwaters to the floodplain of the Jacuí River. In each sampling unit were identified and measured all woody plants with diameter at breast height $\geq 5 \mathrm{~cm}$. From the total sample was performed a cluster analysis of floristic similarity, resulting in three forest communities. These were termed formation, submontane and alluvial, and the area dominated by both formations. For the total sample and the groups was determined the percentage contribution of species and individuals, by classification of ecological guilds of dispersal of seeds and regeneration and geographic quotas. The geographic contingent composition of forest communities was dominated by species of wide distribution. The patterns of ecological guilds were different between groups. However, all groups revealed higher contribution of zoocoric species.
\end{abstract}

Keywords: Forest communities; phytogeography; floristic similarity; forest ecology.

\section{INTRODUÇÃO}

A Depressão Central do Rio Grande do Sul é uma região fisiográfica caracterizada como área de tensão ecológica (LEITE; KLEIN, 1990), em razão da mistura entre as florestas estacionais e os campos. Essas unidades são muito distintas, tanto estrutural quanto floristicamente, havendo claramente espaços onde uma é favorecida em detrimento da outra (MARCHIORI, 2009). 
A presença dessas florestas na região ocorreu somente após a metade do Holoceno, nos últimos quatro mil anos, com as mudanças climáticas do período interglacial (PILLAR; VÉLEZ, 2010). O clima atual da região é úmido e temperado quente, favorável à expansão e domínio das formações florestais e consequente recuo dos campos. O avanço das florestas para o sul do Brasil ocorreu a partir de refúgios remanescentes do longo período xerotérmico da última idade do gelo, no Pleistoceno, de áreas tropicais e subtropicais situadas na região central do país e que avançaram por duas vias de migração, uma litorânea e outra pelo interior do continente (MARCHIORI, 2004). Esses corredores são, respectivamente, o leste Atlântico, recoberto pela Floresta Ombrófila Densa, e o oeste continental, onde predomina a Floresta Estacional das bacias dos rios Paraná e Uruguai. Esses corredores se interpenetram nas encostas e na base do Planalto Sul-brasileiro (JARENKOW; WAECHTER, 2001) e avançam sobre a Depressão Central, pelas calhas dos rios e encostas, necessitando de mais tempo para as florestas dominarem o espaço atual (BEHLING et al., 2005).

Jarenkow e Waechter (2001), tendo conhecimento desse processo de avanço florestal e dos contingentes migratórios das espécies florestais, realizaram a primeira análise qualitativa e quantitativa dos componentes arbóreos de um fragmento florestal localizado entre a Depressão Central e o sopé da Serra Geral, no município de Vale do Sol. A partir desse trabalho, muitos outros estudos semelhantes foram realizados com essa abordagem (BUDKE et al., 2004; LINDENMAIER; BUDKE, 2006; SOARES; FERRER, 2009; SÜHS et al., 2010), sendo a maioria em pequenos fragmentos, além de outros estudos focados nos padrões ecológicos de dispersão de diásporos de espécies arbóreas, estes baseados na morfologia de frutos e sementes (NASCIMENTO et al., 2000; BUDKE et al., 2005; LINDENMAIER; BUDKE, 2006; GIEHL et al., 2007). Contudo, esses trabalhos restringem-se a apenas uma formação florestal, não contemplando o gradiente ambiental dessas formações conectadas na paisagem.

Assim, o presente estudo foi conduzido em uma microbacia na região da Depressão Central, envolvendo fragmentos florestais originados pelo recente avanço migratório de florestas aluviais e submontanas, as quais se encontram em distintas condições ambientais ao longo de um gradiente topográfico. Com isso, a hipótese foi verificar se, entre as Florestas Estacionais Deciduais Aluviais em pequenos córregos e as Florestas Estacionais Deciduais Submontanas, há presença de grupos florísticos independentes de cada formação ou o conjunto destes com outros grupos de influência de ambas as formações, por meio da composição florística, padrões ecológicos de dispersão e regeneração e origem dos contingentes geográficos migratórios das espécies.

\section{MATERIAIS E MÉTODOS}

\section{Área de estudo}

Este estudo foi realizado no Rio Grande do Sul, em uma microbacia localizada na margem direita do baixo rio Jacuí, na região da Depressão Central. A área está inclusa na Fazenda Boa Vista e encontra-se entre os municípios de Pantano Grande e Rio Pardo (Figura 1). O local situa-se em área de transição dos biomas Mata Atlântica e Pampa, compreendido entre as coordenadas geográficas $30^{\circ} 04^{\prime} 55^{\prime \prime} \mathrm{S}$ e $52^{\circ} 25^{\prime} 10^{\prime \prime} \mathrm{O}$ (jusante) a $30^{\circ} 07^{\prime} 16^{\prime \prime} \mathrm{S}$ e $52^{\circ} 22^{\prime} 27^{\prime \prime} \mathrm{O}$ (montante), com altitudes entre 15 a 95 metros. A geologia é formada por sedimentos paleozoicos que fazem parte do rebordo da bacia do Paraná, com predomínio de arenitos médios a finos, siltitos argilosos e lamitos de cores avermelhadas e brancas (SOARES et al., 2008).

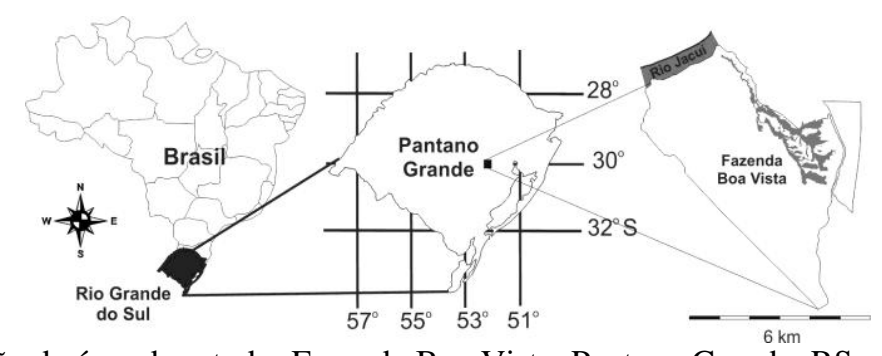

Figura 1. Localização da área de estudo, Fazenda Boa Vista, Pantano Grande, RS. Figure 1. Location of the focused area, Fazenda Boa Vista, Pantano Grande, RS. 
A paisagem é formada por mosaicos de florestas, campos antrópicos e povoamentos florestais de eucaliptos. O clima é classificado como subtemperado úmido (MALUF, 2000) e caracteriza-se por apresentar temperatura média anual de $18,8^{\circ} \mathrm{C}$, variando de $18,1 \mathrm{a} 22^{\circ} \mathrm{C}$. A temperatura média do mês mais frio é menor ou igual a $13{ }^{\circ} \mathrm{C}$, e a do mês mais quente, $24,8{ }^{\circ} \mathrm{C}$. A precipitação fica em torno de $1.400 \mathrm{~mm}$. ano $^{-1}$, tendo um balanço hídrico excedente de zero a $200 \mathrm{~mm}$ e deficiência entre 1 a $150 \mathrm{~mm}$, em curtos períodos de seca no verão. As florestas da região são classificadas como Floresta Estacional Decidual Aluvial e Submontana (INSTITUTO BRASILEIRO DE GEOGRAFIA E ESTATÍSTICA (IBGE), 1986).

\section{Análise da vegetação}

A coleta de dados foi realizada em 40 pontos amostrais, sendo cada um dos pontos constituído por cinco parcelas permanentes de $10 \times 10 \mathrm{~m}\left(100 \mathrm{~m}^{2}\right)$, distribuídos ao longo das vertentes e da planície, incluindo cabeceiras de drenagens, abrangendo diferentes situações geomorfológicas. Em cada unidade de amostra foram identificados e medidos todos os indivíduos lenhosos com diâmetro maior ou igual a $5 \mathrm{~cm}$, medido a $1,30 \mathrm{~m}$ de altura do solo. Esse critério de inclusão também foi adotado para os indivíduos perfilhados, considerando-os como um único indivíduo, desde que um tronco atenda o tamanho de diâmetro na altura do peito. Foram coletados materiais botânicos dos indivíduos arbóreos não identificados em campo e analisados por especialistas, sendo eles incorporados aos herbários do Departamento de Ciências Florestais (HDCF) da Universidade Federal de Santa Maria e da Escola de Florestas de Curitiba (EFC) da Universidade Federal do Paraná. A nomenclatura botânica foi confirmada pela lista da flora do Brasil 2011 (FORZZA et al., 2011) e classificada pelo Sistema Angiosperm Phylogeny Group - APG III (2009).

A diversidade foi avaliada por meio do índice de Shannon ( $\left.\mathrm{H}^{\prime}\right)$ e a equabilidade pelo índice de Pielou (J') (ZAR, 2009). As curvas de acumulação e rarefação de espécies esperadas foram processadas conforme Colwell (2009), sendo a última complementada com curvas de intervalos de confiança 95\% por autorreamostragem. Para obtenção da similaridade florística entre as áreas amostrais, foi feita uma análise de cluster, com dados de densidade das espécies por ponto amostral, tomando por base a distância euclidiana e o método de Ward e realizando-se o cálculo do coeficiente de correlação cofenética, com auxílio do programa computacional PC-ORD for Windows versão 6.0 (MCCUNE; MEFFORD, 2011).

As espécies foram classificadas por categoria do contingente geográfico de migração para o Rio Grande do Sul, por meio da metodologia de Jarenkow e Waechter (2001), com a adaptação de Soares e Ferrer (2009), que consideraram três domínios florestais, sendo eles o Atlântico (leste), o das bacias do Paraná-Uruguai (oeste) e o dos Pinhais (Floresta Ombrófila Mista), além da classe das espécies de ampla distribuição. Para a classificação das espécies, foram utilizadas as listas dos trabalhos pré-citados, além de Budke et al. (2004), Lindenmaier e Budke (2006), Sobral e Jarenkow (2006) e Sühs et al. (2010). As estratégias de dispersão foram divididas em zoocóricas, anemocóricas e autocóricas, de acordo com a morfologia dos frutos e/ou sementes (VAN DER PIJL, 1982). Na classificação da regeneração, as espécies foram qualificadas em pioneiras, clímax exigente de luz e climácicas tolerantes à sombra, conforme Oliveira-Filho et al. (1994).

\section{RESULTADOS E DISCUSSÃO}

\section{Composição e similaridade florística}

Como resultado do levantamento da Floresta Estacional Decidual, Aluvial e Submontana, na fazenda Boa Vista, foram encontradas 90 espécies arbóreas (89 nativas e uma exótica), três arvoretas, um arbusto e duas palmeiras, pertencentes a 67 gêneros e a 37 famílias (Tabela 1). As famílias que apresentaram maior riqueza foram Myrtaceae, com 16 espécies, Salicaceae, com oito espécies, Primulaceae, com seis espécies, Fabaceae, com cinco espécies, Euphorbiaceae, Rubiaceae e Sapotaceae, com quatro espécies cada uma. Essas sete famílias (18,5\% do total) agrupam 47 espécies, ou seja, $49 \%$ da riqueza esperada. Essa composição de famílias é típica da Floresta Estacional, tendo sempre Myrtaceae, Fabaceae e Euphorbiaceae entre as mais diversas, tanto nas florestas mais próximas do contingente Atlântico (JARENKOW; WAECHTER, 2001; BERGAMIN; MONDIN, 2006; MARCHI; JARENKOW, 2008) como nas florestas aluviais (BUDKE et al., 2004; ARAÚJO et al., 2004; BUDKE et al., 2007) ou não aluviais da região central do Rio Grande do Sul (LONGHI et al., 2000; LINDENMAIER; BUDKE, 2006; SCIPIONI et al., 2009; SÜHS et al., 2010) e também nas florestas localizadas mais ao norte do 
estado, na bacia do rio Uruguai, com maior influência do contingente oeste da bacia do Paraná (VACCARO; LONGHI, 1995; SCIPIONI et al., 2011).

Os gêneros mais representativos em número de espécies foram Eugenia, com nove espécies, Myrsine, com seis espécies, Xylosma e Cordia, com três espécies cada. Esses quatro gêneros (6\% do total) reúnem 21 espécies (22\% do total), e os demais 63 gêneros (94\%) agrupam 88\% das espécies. A alta riqueza do gênero Eugenia é fato comum nas florestas da região (LONGHI et al., 2000; JURINITZ; JARENKOW, 2003; ARAÚJO et al., 2004; BUDKE et al., 2004; LINDENMAIER; BUDKE, 2006; SÜHS et al., 2010). Contudo, a riqueza na área de estudo se destaca em comparação com outros trabalhos feitos na região, isso porque neste houve maior intensidade amostral, abrangendo áreas aluviais e submontanas. Por outro lado, a alta riqueza de espécies do gênero Myrsine se deve, em parte, ao fato de as áreas amostrais abrangerem estreitos fragmentos em estágios iniciais a intermediários de sucessão florestal, condição em que as espécies nativas desse gênero se destacam no processo de sucessão (FREITAS; CARRIJO, 2008).

A estimativa da diversidade pelo índice de Shannon (H') para a floresta estudada foi de 3,52 nats/ind. Esse valor foi superior aos estudos citados até o momento para essa mesma unidade, como também em comparação aos valores encontrados e citados por Soares e Ferrer (2009) para Florestas Estacionais Semideciduais no RS. Contudo, esse valor é inferior aos estimados em Florestas Estacionais Semideciduais no norte do Paraná e São Paulo (JURINITZ; JARENKOW, 2003), com área amostral superior a um hectare, e em Florestas Estacionais Aluviais do Bioma Cerrado (SAMPAIO et al., 2000).

Tabela 1. Composição florística do componente arbóreo conforme os grupos de similaridade florística, Floresta Estacional Decidual, Fazenda Boa Vista, RS.

Table 1. Floristic composition of the arboreal component of floristic similarity groups, Deciduous Forest, Boa Vista Farm, RS.

\begin{tabular}{|c|c|c|c|c|c|c|c|}
\hline Família / Nome científico & FB & CG & Dis & $\mathbf{R g}$ & FA & FA/FS & FS \\
\hline \multicolumn{8}{|l|}{ ANACARDIACEAE } \\
\hline Lithrea brasiliensis Marchand & Arv & EAD & Zoo & $\mathrm{P}$ & 3 & 96 & - \\
\hline Lithrea molleoides (Vell.) Engl. & Arv & EAD & Zoo & $\mathrm{P}$ & - & 7 & - \\
\hline Schinus polygamus (Cav.) Cabrera & Arv & EAD & Zoo & $\mathrm{P}$ & - & 2 & - \\
\hline \multicolumn{8}{|l|}{ ANNONACEAE } \\
\hline Annona neosalicifolia $\mathrm{H}$. Rainer & Arv & BPU & Zoo & $\mathrm{CL}$ & 12 & 27 & 14 \\
\hline \multicolumn{8}{|l|}{ AQUIFOLIACEAE } \\
\hline Ilex microdonta Reissek & Arv & PIN & Zoo & CL & - & 13 & 1 \\
\hline \multicolumn{8}{|l|}{ ARECACEAE } \\
\hline Butia odorata (Barb. Rodr.) Noblick \& Lorenzi & Pal & BPU & Zoo & CL & - & 3 & - \\
\hline Syagrus romanzoffiana (Cham.) Glassman & Pal & EAD & Zoo & $\mathrm{CL}$ & 33 & 3 & - \\
\hline \multicolumn{8}{|l|}{ ASTERACEAE } \\
\hline Dasyphyllum spinescens (Less.) Cabrera & Arv & BPU & Ane & $\mathrm{P}$ & - & 10 & - \\
\hline Gochnatia polymorpha (Less.) Cabrera & Arv & EAD & Ane & $\mathrm{P}$ & - & 8 & - \\
\hline \multicolumn{8}{|l|}{ BIGNONIACEAE } \\
\hline Handroanthus heptaphyllus Mattos & Arv & EAD & Ane & CL & - & 6 & 2 \\
\hline \multicolumn{8}{|l|}{ BORAGINACEAE } \\
\hline Cordia americana (L.) Gottschling \& J. S. Mill. & Arv & BPU & Ane & $\mathrm{P}$ & 1 & 26 & 16 \\
\hline Cordia ecalyculata Vell. & Arv & BPU & Zoo & CS & - & - & 2 \\
\hline Cordia trichotoma (Vell.) Arráb. ex Steud. & Arv & EAD & Ane & $\mathrm{P}$ & 3 & 2 & 2 \\
\hline \multicolumn{8}{|l|}{ CANNABACEAE } \\
\hline Celtis iguanaea (Jacq.) Sarg. & Arv & BPU & Zoo & $\mathrm{P}$ & 3 & 6 & 1 \\
\hline Trema micrantha (L.) Blume & Arv & EAD & Zoo & $\mathrm{P}$ & - & 2 & - \\
\hline \multicolumn{8}{|l|}{ CARDIOPTERIDACEAE } \\
\hline Citronella gongonha (Mart.) R. A. Howard & Arv & EAD & Zoo & CL & - & 3 & - \\
\hline Citronella paniculata (Mart.) R. A. Howard & Arv & EAD & Zoo & CL & - & 3 & 1 \\
\hline
\end{tabular}




\begin{tabular}{|c|c|c|c|c|c|c|c|}
\hline \multicolumn{8}{|l|}{ COMBRETACEAE } \\
\hline $\begin{array}{l}\text { Terminalia australis Cambess. } \\
\text { EBENACEAE }\end{array}$ & Arv & EAD & Ane & $\mathrm{P}$ & 18 & - & - \\
\hline $\begin{array}{l}\text { Diospyros inconstans Jacq. } \\
\text { ERYTHROXYLACEAE }\end{array}$ & Arv & BPU & Zoo & $\mathrm{CL}$ & - & 39 & 12 \\
\hline $\begin{array}{l}\text { Erythroxylum deciduum A. St.-Hil. } \\
\text { EUPHORBIACEAE }\end{array}$ & Arv & EAD & Zoo & $\mathrm{P}$ & - & 4 & - \\
\hline Actinostemon concolor (Spreng.) Müll. Arg. & Arv & EAD & Aut & CS & - & 23 & 358 \\
\hline Alchornea triplinervia (Spreng.) Müll. Arg. & Arv & EAD & Zoo & CL & - & - & 1 \\
\hline Sebastiania brasiliensis Spreng. & Arv & EAD & Auto & CL & 175 & 153 & 5 \\
\hline $\begin{array}{l}\text { Sebastiania commersoniana (Baill.) L. B. Sm. \& Downs } \\
\text { FABACEAE }\end{array}$ & Arv & EAD & Auto & $\mathrm{P}$ & 382 & 115 & 14 \\
\hline Calliandra tweedii Benth. & Art & EAD & Zoo & CL & 3 & - & - \\
\hline Dalbergia frutescens (Vell.) Britton & Arv & BPU & Ane & CL & 1 & 2 & - \\
\hline Enterolobium contortisiliquum (Vell.) Morong & Arv & BPU & Zoo & CL & 8 & 11 & 1 \\
\hline Erythrina crista-galli $\mathrm{L}$. & Arv & BPU & Zoo & $\mathrm{P}$ & 7 & 1 & - \\
\hline Inga vera Willd. & Arv & BPU & Zoo & CL & 3 & - & - \\
\hline Lonchocarpus nitidus (Vogel) Benth. & Arv & $\mathrm{BPU}$ & Zoo & CL & 1 & - & - \\
\hline $\begin{array}{l}\text { Machaerium paraguariense Hassl. } \\
\text { LAMIACEAE }\end{array}$ & Arv & BPU & Ane & $\mathrm{CL}$ & 13 & 35 & 4 \\
\hline $\begin{array}{l}\text { Vitex megapotamica (Spreng.) Moldenke } \\
\text { LAURACEAE }\end{array}$ & Arv & EAD & Zoo & CL & 31 & 23 & 2 \\
\hline Nectandra megapotamica (Spreng.) Mez & Arv & EAD & Zoo & CL & - & 39 & 2 \\
\hline Ocotea puberula (Rich.) Nees & Arv & EAD & Zoo & CL & - & - & 1 \\
\hline $\begin{array}{l}\text { Ocotea pulchella (Nees \& Mart.) Mez } \\
\text { LOGANIACEAE }\end{array}$ & Arv & EAD & Zoo & CL & 3 & 38 & 2 \\
\hline $\begin{array}{l}\text { Strychnos brasiliensis Mart. } \\
\text { MALVACEAE }\end{array}$ & Arv & EAD & Zoo & CL & 1 & - & - \\
\hline $\begin{array}{l}\text { Luehea divaricata Mart. \& Zucc. } \\
\text { MELASTOMATACEAE }\end{array}$ & Arv & EAD & Ane & CL & 35 & 44 & 16 \\
\hline $\begin{array}{l}\text { Miconia hyemalis A. St.-Hil. \& Naudin } \\
\text { MELIACEAE }\end{array}$ & Arb & EAD & Zoo & $\mathrm{P}$ & - & 1 & - \\
\hline Trichilia claussenii C. DC. & Arv & BPU & Zoo & CS & - & 38 & 24 \\
\hline $\begin{array}{l}\text { Trichilia elegans A. Juss. } \\
\text { MORACEAE }\end{array}$ & Arv & BPU & Zoo & $\mathrm{CS}$ & 1 & 13 & - \\
\hline Ficus cestrifolia Schott ex Spreng. & Arv & ATL & Zoo & $\mathrm{CL}$ & - & 1 & 2 \\
\hline Ficus luschnathiana (Miq.) Miq. & Arv & EAD & Zoo & CL & 1 & 3 & 1 \\
\hline $\begin{array}{l}\text { Sorocea bonplandii (Baill.) W. C. Burger et al. } \\
\text { MYRTACEAE }\end{array}$ & Arv & EAD & Zoo & CS & - & 32 & 42 \\
\hline Blepharocalyx salicifolius (Kunth) O. Berg & Arv & EAD & Zoo & CL & 4 & 12 & 1 \\
\hline Campomanesia xanthocarpa (Mart.) O. Berg & Arv & EAD & Zoo & CS & 3 & 24 & 3 \\
\hline Eugenia hiemalis Cambess. & Arv & ATL & Zoo & CL & 10 & 11 & 1 \\
\hline Eugenia involucrata DC. & Arv & BPU & Zoo & CL & 1 & 1 & 1 \\
\hline Eugenia pluriflora DC. & Art & PIN & Zoo & CL & 1 & - & - \\
\hline Eugenia rostrifolia D. Legrand & Arv & BPU & Zoo & CL & 2 & - & - \\
\hline Eugenia speciosa Cambess. & Arv & ATL & Zoo & CS & 4 & - & - \\
\hline Eugenia uniflora L. & Arv & EAD & Zoo & CL & 104 & 40 & - \\
\hline Eugenia uruguayensis Cambess. & Arv & EAD & Zoo & $\mathrm{CL}$ & 60 & 15 & - \\
\hline
\end{tabular}




\begin{tabular}{|c|c|c|c|c|c|c|c|}
\hline Eugenia verticillata (Vell.) Angely & Art & ATL & Zoo & $\mathrm{CS}$ & - & 5 & 11 \\
\hline Eugenia ramboi D. Legrand & Arv & BPU & Zoo & $\mathrm{CS}$ & 2 & - & - \\
\hline Myrcia multiflora (Lam.) DC. & Arv & ATL & Zoo & $\mathrm{CL}$ & 37 & 1 & - \\
\hline Myrcia palustris DC. & Arv & EAD & Zoo & $\mathrm{CL}$ & 3 & 1 & - \\
\hline Myrcianthes pungens (O. Berg) D. Legrand & Arv & BPU & Zoo & $\mathrm{CL}$ & - & 54 & 27 \\
\hline Myrciaria tenella (DC.) O. Berg & Arv & EAD & Zoo & $\mathrm{CS}$ & 59 & 1 & - \\
\hline Myrrhinium atropurpureum Schott & Arv & EAD & Zoo & $\mathrm{CL}$ & 1 & 2 & - \\
\hline \multicolumn{8}{|l|}{ OLEACEAE } \\
\hline Chionanthus filiformis (Vell.) P. S. Green & Arv & ATL & Zoo & CS & - & 3 & 1 \\
\hline \multicolumn{8}{|l|}{ POLYGONACEAE } \\
\hline Coccoloba cordata Cham. & Arv & EAD & Zoo & $\mathrm{CL}$ & 9 & 2 & - \\
\hline Ruprechtia laxiflora Meisn. & Arv & EAD & Ane & $\mathrm{CL}$ & 4 & 2 & - \\
\hline \multicolumn{8}{|l|}{ PRIMULACEAE } \\
\hline Myrsine coriacea (Sw.) R. Br. ex Roem. \& Schult. & Arv & EAD & Zoo & $\mathrm{P}$ & - & 3 & - \\
\hline Myrsine guianensis (Aubl.) Kuntze & Arv & EAD & Zoo & $\mathrm{CL}$ & - & 2 & - \\
\hline Myrsine laetevirens (Mez) Arechav. & Arv & BPU & Zoo & $\mathrm{P}$ & 1 & 18 & - \\
\hline Myrsine loefgrenii (Mez) Imkhan. & Arv & BPU & Zoo & $\mathrm{CL}$ & - & 13 & 2 \\
\hline Myrsine umbellata Mart. & Arv & EAD & Zoo & $\mathrm{CL}$ & - & 13 & 2 \\
\hline Myrsine parvula (Mez) Otegui & Arv & EAD & Zoo & $\mathrm{P}$ & - & 3 & - \\
\hline \multicolumn{8}{|l|}{ RHAMNACEAE } \\
\hline Scutia buxifolia Reissek & Arv & BPU & Zoo & $\mathrm{CL}$ & 5 & 9 & - \\
\hline \multicolumn{8}{|l|}{ ROSACEAE } \\
\hline Prunus myrtifolia (L.) Urb. & Arv & EAD & Zoo & $\mathrm{CL}$ & - & 3 & - \\
\hline \multicolumn{8}{|l|}{ RUBIACEAE } \\
\hline Chomelia obtusa Cham. \& Schltdl. & Arv & EAD & Zoo & $\mathrm{CL}$ & 6 & 50 & 16 \\
\hline Faramea montevidensis (Cham. \& Schltdl.) DC. & Arv & ATL & Zoo & $\mathrm{CS}$ & - & 8 & 22 \\
\hline Guettarda uruguensis Cham. \& Schltdl. & Arv & BPU & Zoo & $\mathrm{CL}$ & 46 & 7 & - \\
\hline Randia ferox (Cham. \& Schltdl.) DC. & Arv & EAD & Zoo & $\mathrm{CS}$ & 1 & 4 & - \\
\hline \multicolumn{8}{|l|}{ RUTACEAE } \\
\hline Citrus sp. & Arv & EXO & Zoo & INV & - & 2 & 1 \\
\hline Zanthoxylum fagara (L.) Sarg. & Arv & BPU & Zoo & $\mathrm{P}$ & - & 6 & - \\
\hline Zanthoxylum rhoifolium Lam. & Arv & EAD & Zoo & $\mathrm{P}$ & - & 16 & 2 \\
\hline \multicolumn{8}{|l|}{ SALICACEAE } \\
\hline Banara parviflora (A. Gray) Benth. & Arv & EAD & Zoo & $\mathrm{CL}$ & 1 & 2 & 2 \\
\hline Banara tomentosa $\mathrm{Clos}$ & Arv & BPU & Zoo & $\mathrm{CS}$ & - & 5 & 3 \\
\hline Casearia decandra Jacq. & Arv & BPU & Zoo & $\mathrm{CS}$ & 8 & 49 & 13 \\
\hline Casearia sylvestris $\mathrm{Sw}$. & Arv & EAD & Zoo & $\mathrm{CL}$ & 53 & 115 & 16 \\
\hline Salix humboldtiana Willd. & Arv & EAD & Ane & $\mathrm{P}$ & 3 & - & - \\
\hline Xylosma prockia (Turcz.) Turcz. & Arv & EAD & Zoo & $\mathrm{CL}$ & - & 1 & - \\
\hline Xylosma pseudosalzmannii Sleumer & Arv & EAD & Zoo & CS & 1 & 1 & - \\
\hline Xylosma tweediana (Clos) Eichler & Arv & BPU & Zoo & $\mathrm{CL}$ & - & 14 & 1 \\
\hline \multicolumn{8}{|l|}{ SAPINDACEAE } \\
\hline Allophylus edulis (A. St.-Hil. et al.) Hieron. ex Niederl. & Arv & EAD & Zoo & $\mathrm{CL}$ & 34 & 84 & 2 \\
\hline Cupania vernalis Cambess. & Arv & EAD & Zoo & $\mathrm{CL}$ & - & 7 & 3 \\
\hline Matayba elaeagnoides Radlk. & Arv & EAD & Zoo & $\mathrm{CL}$ & 4 & 21 & 3 \\
\hline \multicolumn{8}{|l|}{ SAPOTACEAE } \\
\hline Chrysophyllum gonocarpum (Mart. \& Eichler ex Miq.) Engl. & Arv & BPU & Zoo & $\mathrm{CS}$ & - & 1 & 6 \\
\hline Chrysophyllum marginatum (Hook. \& Arn.) Radlk. & Arv & BPU & Zoo & $\mathrm{CL}$ & 28 & 141 & 18 \\
\hline
\end{tabular}




\begin{tabular}{llllcc}
\hline $\begin{array}{l}\text { Pouteria gardneriana (A. DC.) Radlk. } \\
\text { Sideroxylon obtusifolium (Roem. \& Schult.) T. D. Penn. }\end{array}$ & Arv BPU & Zoo & CL & 10 & - \\
SOLANACEAE & Arv EAD Zoo & CL & - & 5 & - \\
$\begin{array}{l}\text { Solanum pseudoquina A. St.-Hil. } \\
\text { Solanum sanctae-catharinae Dunal }\end{array}$ & Arv ATL Zoo & CL & - & 1 & 2 \\
SYMPLOCACEAE & Arv EAD Zoo & CL & - & 1 & - \\
$\begin{array}{l}\text { Symplocos uniflora (Pohl) Benth. } \\
\text { VERBNACEAE }\end{array}$ & Arv EAD Zoo & CS & - & 2 & - \\
Citharexylum montevidense (Spreng.) Moldenke & & & & & \\
\hline
\end{tabular}

FB: forma biológica (Arv: árvore; Art: arvoreta; Arb: arbusto; Pal: palmeira); CG: contingente geográfico (EAD: espécie de ampla distribuição; BPU: espécie das bacias dos rios Paraná e Uruguai; ATL: espécie do contingente Atlântico; PIN: espécie dos pinhais; EXO: espécie exótica); Disp: Forma de dispersão (Zoo: zoocoria; Ane: anemocoria; Aut: autocoria); Rg: regeneração (CL: clímax exigente em luz; CS: clímax tolerante à sombra; P: pioneira); INV: invasora.

A alta riqueza e diversidade é o resultado da elevada heterogeneidade ambiental, associada às condições aluviais e montanas e à presença de trechos florestais entre estas com diferentes estágios sucessionais secundários. A curva de acumulação de espécies (linha cinza), com os pontos amostrais ordenados de forma decrescente da encosta para a planície, informa sobre isso, conforme as transposições abruptas visíveis na figura 2. Contudo, com base na curva de acumulação de espécies (Figura 2), estimouse que a amostra contemplou $95,8 \%$ das espécies esperadas para a comunidade estudada.

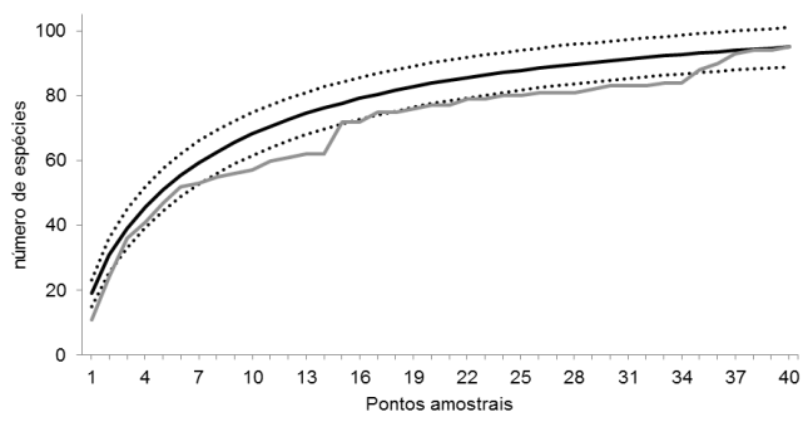

Figura 2. Curvas de acumulação de espécies: linha cinza representa a sequência de amostragem ordenando os pontos amostrais de forma decrescente no gradiente topográfico; linha preta, a autorreamostragem com os seus limites de intervalo de confiança (linhas tracejadas).

Figure 2. Species accumulation curves: gray line represent the sampling by sample points, descending order in topographic gradient; black line, bootstraps with their limits of confidence intervals (dashed lines).

Na curva de acumulação de espécies, é constatado um acréscimo de novas espécies no ponto amostral 15 , com dez espécies $(16,1 \%)$ em posição intermediária na encosta. Nessa mesma curva, podemse identificar novos acréscimos específicos a partir do ponto amostral 35 (com um acréscimo de quatro espécies) e no final da amostragem, já nas proximidades da floresta ciliar do rio Jacuí, onde a floresta apresenta condições ambientais e estágios sucessionais secundários distintos, influenciando, assim, o comportamento da curva.

A presença de duas formações florestais em ambientes distintos na área e mais a existência de mudanças abruptas na curva de acumulação sugerem a presença de grupos florísticos distintos, que foram estruturados por meio da análise de agrupamento (Figura 3) e analisados separadamente (Tabela 1). O valor do coeficiente de correlação cofenética foi de 0,352 . Desse modo, ao corte de $0,30 \%$ da informação remanescente no dendrograma, foram definidos três grupos, nomeados de formação submontana, formação aluvial e o último com o termo aluvial/submontana, em razão de apresentar componentes florísticos das duas formações e predominando em posições intermediárias no gradiente topográfico. 


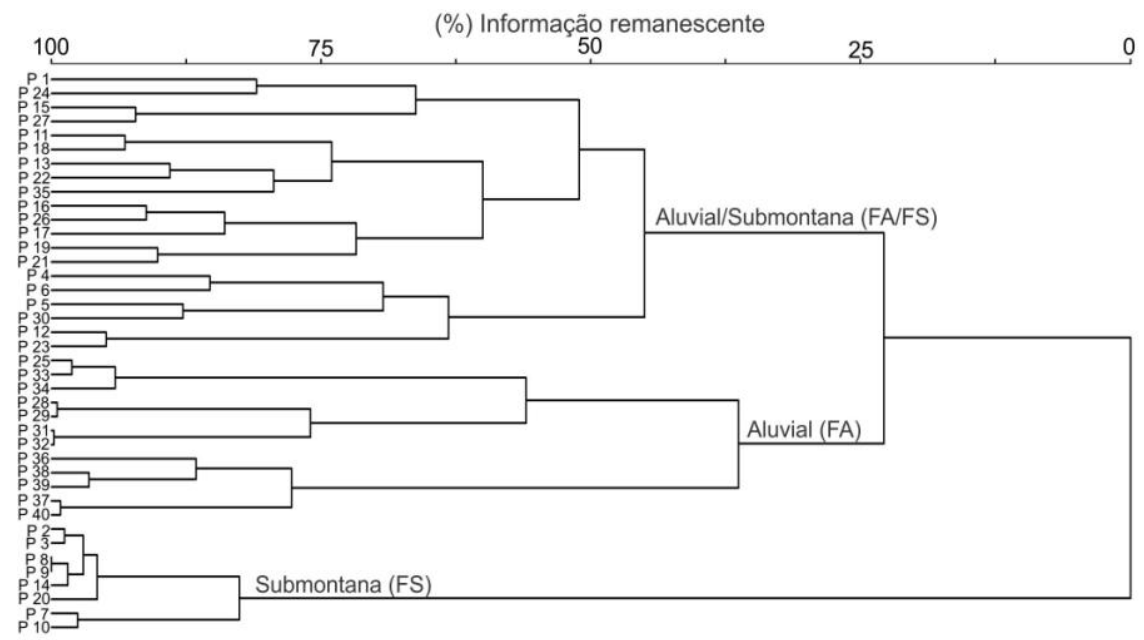

Figura 3. Dendrograma de similaridade florística dos pontos amostrais, Fazenda Boa Vista, Pantano Grande, RS.

Figura 3. Dendrogram of floristic of the sample points, Boa Vista Farm, Pantano Grande, RS.

Com a análise, foi possível diferenciar as formações aluvais e submontanas típicas em estágios secundários avançados nos extremos do gradiente e as áreas representadas por ambas as formações (FA/FS), abrangendo a maioria dos pontos amostrais em estreitos fragmentos de floresta ou em locais próximos de domínio das formações em estágios secundários iniciais e intermediários de sucessão condicionados por distúrbios antrópicos e erosivos de canalização. De acordo com essa segregação, no grupamento da formação aluvial (FA) foram encontradas 53 espécies e 34,7\% dos indivíduos da amostra total. Por sua vez, 49 espécies e 19,1\% dos indivíduos foram amostrados na formação submontana (FS), havendo 82 espécies e 46,2\% dos indivíduos nas áreas de influência de ambas as formações (FA/FS). Entre essas espécies, 11 foram exclusivas de FA, três de FS e 18 de FA/FS. Vinte e quatro espécies foram comuns a todos os grupos, havendo 18 espécies compartilhadas entre FA/FS e FA, além de 22 espécies compartilhadas entre FA/FS e FS. Nenhuma espécie foi compartilhada entre FS e FA (Figura 4).

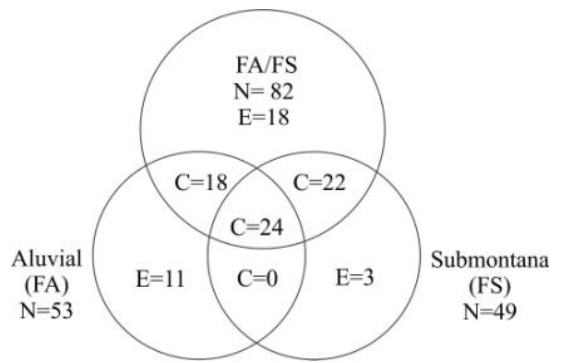

Figura 4. Diagrama de Venn discriminando os números de espécies comuns e exclusivas entre os grupos florestais identificados em fragmento amostrada na Fazenda Boa Vista, Pantano Grande, RS. E: espécies exclusivas; C: espécies comuns.

Figure 4. Distribution of species among sampling areas separated by the floristic similarity, Boa Vista Farm, Pantano Grande, RS. E: exclusive species; C: common species.

\section{Contingente geográfico e padrões ecológicos}

Considerando a abundância encontrada, o contigente geográfico migratório foi dominado por espécies classificadas de ampla distribuição. Esse grupo representa 70,2\% dos indivíduos amostrados e $56,8 \%$ das espécies na amostra geral. Aqueles indivíduos classificados como pertencentes ao contingente florístico da bacia do Paraná e Uruguai somam $26 \%$ do total e $32,6 \%$ das espécies, enquanto o contingente do corredor Atlântico foi representado por 3,4\% de indivíduos amostrados e 8,4\% das 
espécies. A minoria dos indivíduos amostrados foi classificada entre aqueles oriundos do contingente dos Pinhais $(0,4 \%)$, os quais foram representados por somente duas espécies. As proporções de indivíduos e espécies de cada um desses contingentes de acordo com os grupos formados foram semelhantes à análise geral, bem como quando comparados entre si (Figura 5).

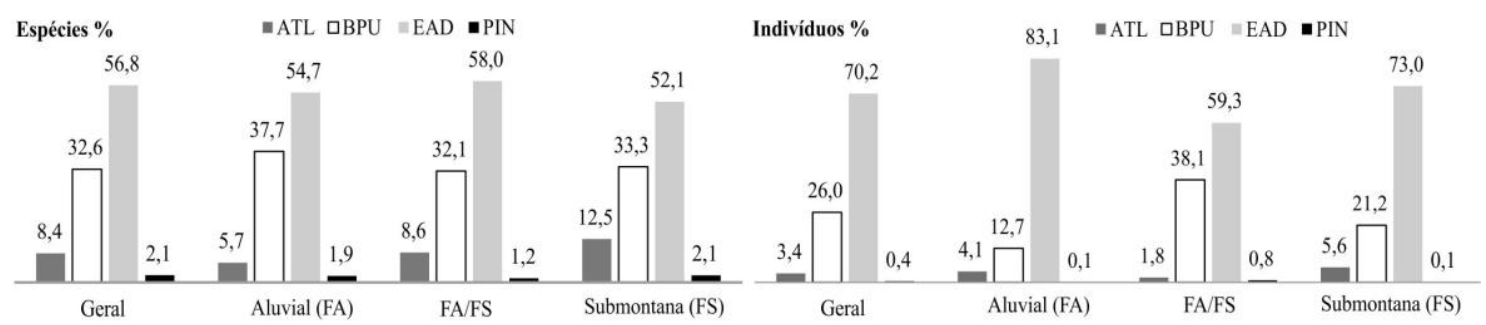

Figura 5. Distribuição da frequência das espécies e dos indivíduos por contingentes geográficos considerando os grupos de similaridade florística (Aluvial - FA; Aluvial/Submontano - FA/FS; Submontano - FS) e dados totais, Pantano Grande, RS. ATL: Atlântico; BUP: Bacia do Uruguai e Paraguai; EAP: espécies de ampla dispersão; PIN: pinhais.

Figure 5. Representation of the percentage contribution of species and individuals by geographic quotas in floristic similarity groups and the general sample, Pantano Grande, RS. ATL: Atlantic; BUP: River Basins of Uruguay and Paraguay; EAP: species of wide dispersion; PIN: Araucaria Forest.

Esses resultados também são parecidos aos encontrados por Budke et al. (2004) mais a oeste da área deste estudo, em uma floresta aluvial (Santa Maria), e por Sühs et al. (2010) na Serra Geral, ao norte da área do estudo, ambos com maior contribuição de indivíduos de ampla distribuição. Mas o resultado encontrado neste estudo foi diferente em comparação aos trabalhos em florestas secundárias em áreas não aluviais, no município de Cachoeira do Sul (LINDENMAIER; BUDKE, 2006) e na Serra Geral a oeste, por Scipioni et al. (2009), nos quais houve maior contribuição de espécies e de indivíduos do contingente migratório oeste.

Por outro lado, Jarenkow e Waechter (2001) observaram a leste na Serra Geral uma maior representação do contingente migratório leste, com $18,2 \%$ das espécies e $29,9 \%$ dos indivíduos maiores e dos indivíduos migratórios de ampla dispersão $(55,2 \%)$, contra a menor densidade dos indivíduos do contingente migratório oeste $(14,9 \%)$. Todos esses resultados comprovam, em parte, a influência da proximidade geográfica aos corredores migratórios de Waechter e Jarenkow (2001) na composição florística das comunidades vegetais. Porém a contribuição de espécies de ampla distribuição é o principal fator que condiciona a variação de importância entre os contingentes geográficos migratórios nas Florestas Estacionais Deciduais, pela sua maior diversidade de espécies e contribuição de indivíduos. Isso pode ser considerado um argumento em favor do recente avanço das florestas nas áreas deste estudo, em razão de baixa contribuição de indivíduos típicos predominantes dos contingentes geográficos migratórios oeste $(26,0 \%)$ e leste $(3,4 \%)$, favorecendo as espécies de ampla dispersão.

$\mathrm{Na}$ análise das estratégias de dispersão, a amostragem geral indicou maior riqueza de espécies $(85,3 \%$; $81 \mathrm{spp}$.) e maior número de indivíduos zoocóricos $(58,7 \% ; 2.104$ ind.), como pode ser visto na figura 6. O valor superior a $80 \%$ de espécies zoocóricas foi encontrado para os três grupos, o que, de acordo com Liebsch et al. (2008), caracteriza as florestas tropicais atlânticas como maduras, tendo idades superiores a 60 anos, assemelhando-se às condições desse estudo. A dominância dessa forma de dispersão destaca-se frente às demais em Floresta Estacional na região (BUDKE et al., 2005; LINDENMAIER; BUDKE, 2006; GIEHL et al., 2007; NASCIMENTO et al., 2000), bem como também em Florestas Estacionais secundárias estudadas em Minas Gerais (NUNES et al., 2003; PEREIRA et al., 2010).

Embora a dispersão autocórica seja representada por somente três espécies (todas Euphorbiaceae), essa síndrome abriga um grande número de indivíduos (Figura 6 A). Na formação aluvial, as duas espécies do gênero Sebastiania representam 44,7\% da densidade, reduzindo significativamente a proporção zoocórica para 49\% (Figura 6 B). Esse resultado foi o mesmo encontrado 
por Budke et al. (2005) em floresta aluvial, com alta abundância dessas mesmas espécies da família Euphorbiaceae encontradas neste estudo.
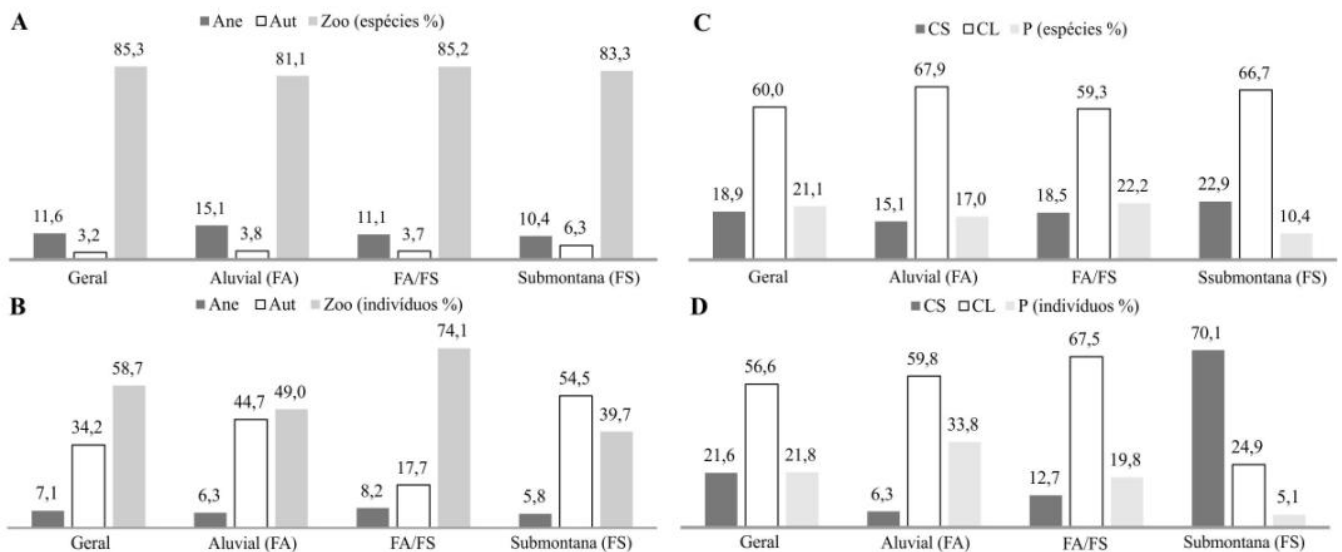

Figura 6. A contribuição das espécies (A) e dos indivíduos (B) por guildas ecológicas de dispersão, espécies (C) e indivíduos (D) por guildas ecológicas de regeneração, nos grupos de similaridade florística e amostragem geral, Pantano Grande, RS. Ane: anemocoria; Aut: autocoria; Zoo: zoocoria; CS: clímax tolerante a sombra; CL: clímax exigente em luz; P: pioneira.

Figure 6. The contribution of species (A) and (B) individuals by ecological guilds of seed dispersal, (C) species and (D) individuals by ecological guilds of regeneration, groups in floristic and general sampling, Pantano Grande, RS. Ane: anemochory; Aut:: autochory; Zoo: zoochory; CS: the shade tolerant climax; CL: light-demanding climax; P: pioneer.

$\mathrm{Na}$ formação submontana, há predominância da síndrome de dispersão autocórica, diferenciando-se dos demais agrupamentos, em razão do grande número de indivíduos de Actinostemon concolor, espécie típica do sub-bosque. Segundo Liebsch et al. (2008), a alta contribuição de indivíduos do sub-bosque é característica de florestas mais velhas ou de estágio sucessional mais avançado, o que permite diferenciar a formação submontana das formações aluviais citadas anteriormente, acompanhando os trabalhos de Lindenmaier e Budke (2006) e Giehl et al. (2007), nos quais a dispersão anemocórica está em segunda posição de importância em densidade relativa e diversidade.

Conforme Giehl et al. (2007), as diferenças locais no espectro de dispersão são esperadas, devido às variações na composição florística, que podem ser condicionadas pelo ambiente, pelo estágio de sucessão e pelos distúrbios antrópicos ou naturais. Como grande parte dessas causas tem efeito e não foram mensuradas em grande parte das pesquisas citadas, torna-se impraticável traçar com maior detalhamento a origem das diferenças entre elas, o que é agravado também pela falta de um histórico preciso das áreas estudadas.

$\mathrm{Na}$ figura 6 estão representadas as guildas de regeneração e verifica-se que as espécies climácicas são as mais importantes, agregando maior quantidade de indivíduos e maior diversidade, tanto para os grupos quanto para a amostra geral. A exceção é constituída pelo grupo da formação submontana, com $70,1 \%$ dos indivíduos enquadrados como sendo clímax tolerante a sombra, seguidos de $24,9 \%$ como clímax exigente de luz e 5,1\% como pioneira. Esse resultado é interessante quando se comparam a contribuição dos indivíduos pioneiros e clímax tolerantes a sombra entre os três agrupamentos, pois nessas classes são inversamente proporcionais quando se visualiza o gradiente ambiental, com o grupo FA/FS apresentando valores intermediários de contribuição. O maior número de indivíduos pioneiros é encontrado na formação aluvial $(33,8 \%)$, seguida pelo grupo FA/FS $(19,8 \%)$. O menor valor de indivíduos pioneiros foi encontrado na formação submontana, com 5,1\%. O valor de indivíduos da categoria clímax tolerante a sombra é máximo na formação submontana, decrescendo para $12,7 \%$ no grupo FA/FS e aproximando-se de 6,3\% na formação aluvial (Figura 6 C e D).

Os maiores percentuais de indivíduos pioneiros na formação aluvial frente às demais situações do estudo podem estar ligados aos diferentes processos de sedimentação pelos pulsos de inundações e aos 
distúrbios de erosão que originam os canais de drenagem no ambiente aluvial (JOHNSON, 1994; OSTERKAMP; HUPP, 2010), como também ao maior efeito de borda na interface canais/fragmento, pela presença de canais de maior dimensão. Nas florestas de encosta, os distúrbios são baixos e os canais são menores ou ausentes, possibilitando um dossel fechado e um processo de sucessão contínuo, com estratificação, onde há um maior número de indivíduos no sub-bosque e árvores emergentes, em comparação com a floresta aluvial.

\section{CONCLUSÕES}

O contingente geográfico de ocorrência das espécies e indivíduos na amostragem total e nos grupos florísticos obtidos foi dominado pelo contingente de ampla distribuição, seguido pela bacia do Paraná e Uruguai, Atlântico e pelos Pinhais. A partir da análise desses grupos florísticos, observou-se que a distribuição das espécies nessas florestas apresentou distintos padrões de compartilhamento, destacando-se a não constatação de espécies comuns em ambos os grupos situados nos extremos do gradiente, o que demonstra um trânsito obrigatório das espécies por meio do grupo FA/FS. Essas diferenças também foram detectadas pelas guildas ecológicas de dispersão de diásporos pela classificação por espécies e abundâncias nessas diferentes tipologias. A submontana (grupo) apresentou maior contribuição de indivíduos do sub-bosque, climácicos de sombra e autocóricos, enquanto a aluvial e o grupo FA/FS apresentaram maior contribuição de indivíduos pioneiros e climácicos exigentes em luz, menor contribuição do sub-bosque e maior percentagem de indivíduos zoocóricos. Apesar dessas diferenças, todos os grupos tiveram a maior contribuição da diversidade de espécies zoocóricas, demonstrando a importância dessas florestas para a fauna local.

\section{REFERÊNCIAS}

ANGIOSPERM PHYLOGENY GROUP (APG) III. An update of the Angiosperm Phylogeny Group classification for the orders and families of flowering plants: APG III. Botanical Journal of the Linnean Society, Londres, v. 161, p. 105 - 121, 2009.

ARAÚJO, M. M.; LONGHI, S. J.; BRENA, D. A.; BARROS, P. L.; FRANCO, S. Análise de agrupamento da vegetação de um fragmento de Floresta Estacional Decidual Aluvial, Cachoeira do Sul, RS, Brasil. Ciência Florestal, Santa Maria, v. 14, n. 1, p. 133 - 147, 2004.

BEHLING, H.; PILLAR, V. D. P.; BAUERMANN, S. G. Late quaternary grassland (Campos), gallery forest, fire and climate dynamics, studied by pollen, charcoal and multivariate analysis of the São Francisco de Assis core in western Rio Grande do Sul (southern Brazil). Review of Palaeobotany and Palynology, v. 133, p. 235 - 248, 2005.

BERGAMIN, R. S.; MONDIN, C. A. Composição florística e relações fitogeográficas do componente arbóreo de um fragmento florestal no município de Barra do Ribeiro, Rio Grande do Sul, Brasil. Pesquisas. Botânica, São Leopoldo, v. 57, p. 217 - 230, 2006.

BUDKE, J. C.; GIEHL, E. L. H.; ATHAYDE, E. A.; EISINGER, S. M.; ZÁCHIA, R. A. Florística e fitossociologia do componente arbóreo de uma floresta ribeirinha, arroio Passo das Tropas, Santa Maria, RS, Brasil. Acta Bot. Bras., São Paulo, v. 18, n. 3, p. 581 - 589, 2004.

BUDKE, J. C.; ATHAYDE, E. A.; GIEHL, E. L. H.; ZÁCHIA, R. A.; EISINGER, S. M. Composição florística e estratégias de dispersão de espécies lenhosas em uma floresta ribeirinha, arroio Passo das Tropas, Santa Maria, RS, Brasil. Iheringia, Sér. Bot., Porto Alegre, v. 60, n. 1, p. 17 - 24, 2005.

BUDKE, J. C.; JARENKOW, J. C.; OLIVEIRA-FILHO, A. T. Relationships between tree component structure, topography and soils of a riverine forest, rio Botucaraí, southern Brazil. Plant Ecol, v. 189, p. 187 - 200, 2007.

COLWELL, R. K. EstimateS: statistical estimation of species richness and shared species from samples. Version 8.2. User's Guide and application, 2009. Disponível em: 〈http://purl.oclc.org/estimates〉. Acesso em: 17/02/2012. 
FORZZA, R. C. et al. Lista da Flora do Brasil 2011. Jardim Botânico do Rio de Janeiro. Disponível em: <http://floradobrasil.jbrj.gov.br/2011//>. Acesso em: 09/12/2011.

FREITAS, M. de F.; CARRIJO, T. T. A família Myrsinaceae nos contrafortes do maciço da Tijuca e entorno do Jardim Botânico do Rio de Janeiro, Brasil. Rodriguésia, Rio de Janeiro, v. 59, n. 4, p. 813 $828,2008$.

GIEHL, E. L. H.; ATHAYDE, E. A.; BUDKE, J. C.; GESING, J. P. A.; EISINGER, S. M.; CANTO DOROW, T. S. Espectro e distribuição vertical das estratégias de dispersão de diásporos do componente arbóreo em uma Floresta Estacional no sul do Brasil. Acta Bot. Bras., São Paulo, v. 21, n. 1, p. 137 145, 2007.

INSTITUTO BRASILEIRO DE GEOGRAFIA E ESTATÍSTICA (IBGE). Folha SH.22 Porto Alegre e partes das Folhas SH. 21 Uruguaiana e SI. 22 Lagoa Mirim: geologia, geomorfologia, pedologia, vegetação, uso potencial da terra. Fundação Instituto Brasileiro de Geografia e Estatística. Rio de Janeiro: IBGE, 1986. (Levantamento dos Recursos Naturais, v. 33)

JARENKOW, J. A.; WAECHTER, J. Composição, estrutura e relações florísticas do componente arbóreo de uma Floresta Estacional no Rio Grande do Sul, Brasil. Rev. Bras. Bot., São Paulo, v. 24, n. 3, p. 263 $272,2001$.

JOHNSON, W. C. Woodland expansion in the Platte river, Nebraska: patterns and causes. Ecological Monographs, Lawrence, v. 64, n. 1, p. 45 - 84, 1994.

JURINITZ, C. F.; JARENKOW, J. A. Estrutura do componente arbóreo de uma Floresta Estacional na Serra do Sudeste, Rio Grande do Sul, Brasil. Revista Brasileira de Botânica, São Paulo, v. 26, n. 4, p. 475 - 487, 2003.

LEITE, P. F.; KLEIN, R. M. Vegetação. In: Geografia do Brasil: Região Sul. Rio de Janeiro: Instituto Brasileiro de Geografia e Estatística, IBGE, 1990. v. 2, p. 113 - 150.

LIEBSCH, D.; MARQUES, M. C. M.; GOLDENBERG, R. How long does the Atlantic Rain Forest take to recover after a disturbance? Changes in species composition and ecological features during secondary succession. Biological Conservation, Essex, v. 141, p. 1717 - 1725, 2008.

LINDENMAIER, D. de S.; BUDKE, J. C. Florística, diversidade e distribuição espacial das espécies arbóreas em uma Floresta Estacional na Bacia do Rio Jacuí, Sul do Brasil. Pesquisas, Botânica, São Leopoldo, n. 57, p. 193 - 216, 2006.

LONGHI, S. J.; ARAÚJO, M. M.; KELLING, M. B.; HOPPE, J. M.; MÜLLER, I.; BORSOI, G. A. Aspectos fitossociológicos de fragmentos de Floresta Estacional Decidual, Santa Maria, RS. Ciência Florestal, Santa Maria, v. 10, n. 2, p. 59 - 74, 2000.

MALUF, J. R. T. Nova classificação climática do Estado do Rio Grande do Sul. Revista Brasileira de Agrometeorologia, Santa Maria, v. 8, n. 1, p. 141 - 150, 2000.

MARCHI, T. C. de; JARENKOW, J. A. Estrutura do componente arbóreo de mata ribeirinha no rio Camaquã, município de Cristal, Rio Grande do Sul, Brasil. Iheringia, Sér. Bot., Porto Alegre, v. 63, n. 2, p. $241-248,2008$.

MARCHIORI, J. N. C. Fitogeografia do Rio Grande do Sul: Campos Sulinos. Porto Alegre: EST Edições, 2004. 110 p.

MARChIORI, J. N. C. A vegetação em Santa Maria. Ciência \& Ambiente, Santa Maria, n. 38, p. 93 $112,2009$.

McCUNE, B.; MEFFORD, M. J. PC-ORD: multivariate analysis of ecological data.version 6.0. Gleneden Beach, Oregon, U.S.A.: MjM Software, 2011. 
NASCIMENTO, A. R. T.; LONGHI, S. J.; ALVAREZ FILHO, A.; GOMES, G. S. Análise da diversidade florística e dos sistemas de dispersão de sementes em um fragmento florestal na região central do estado do RS. Napaea, Porto Alegre, RS, v. 12, p. 49 - 67, 2000.

NUNES, Y. R. F.; MENDONÇA, A. V. R.; BOTEZELLI, L.; MACHADO, E. L. M.; OLIVEIRAFILHO, A. T. Variações da fisionomia, diversidade e composição de guildas da comunidade arbórea em um fragmento de Floresta Semidecidual em Lavras, MG. Acta Bot. Bras., São Paulo, v. 17, n. 2, p. 213 229, 2003.

OLIVEIRA-FILHO, A. T.; VILELA, E.; CARVALHO, D. A.; GAVILANES, M. L. Effects of soils and topography on the distribution of tree species in a tropical riverine forest in south-eastern Brazil. Journal of Tropical Ecology, Cambridge, v. 10, p. 483 - 508, 1994.

OSTERKAMP, W. R.; HUPP, C. R. Fluvial processes and vegetation - glimpses of the past, the present, and perhaps the future. Geomorphology, Amsterdam, v. 116, p. 274 - 285, 2010.

PEREIRA, I. M.; BOTELHO, S. A.; VAN DEN BERG, E.; OLIVEIRA-FILHO, A. T.; MACHADO, E. L. M. Caracterização ecológica de espécies arbóreas ocorrentes em ambientes de mata ciliar, como subsídio à recomposição de áreas alteradas nas cabeceiras do rio Grande, Minas Gerais, Brasil. Ciência Florestal, Santa Maria, v. 20, n. 2, p. 235 - 253, 2010.

PILLAR, V. de P.; VÉLEZ, E. Extinção dos Campos Sulinos em Unidades de Conservação: um fenômeno natural ou um problema ético? Natureza \& Conservação, v. 8, n. 1, p. 84 - 86, 2010.

SAMPAIO, A. B.; WALTER, B. T. M. T.; FELFILI, J. M. Diversidade e distribuição de espécies arbóreas em duas matas de galeria na microbacia do Riacho Fundo, Distrito Federal. Acta Bot. Bras., São Paulo, v. 14, n. 2, p. 197 - 214, 2000.

SCIPIONI, M. C.; LONGHI, S. J.; ARAÚJO, M. M.; REINERT, D. J. Regeneração natural de um fragmento da Floresta Estacional Decidual na reserva biológica do Ibicuí-Mirim (RS). Floresta, Curitiba, v. 39, n. 3, p. 675 - 690, 2009.

SCIPIONI, M. C.; FINGER, C. A. G.; CANTARELlI, E. B.; DENARDI, L.; MEYER, E. A. Fitossociologia em fragmento florestal no noroeste do estado do Rio Grande do Sul. Ciência Florestal, Santa Maria, v. 21, n. 3, p. 407 - 417, 2011.

SOARES, L. R.; FERRER, R. S. Estrutura do componente arbóreo em uma área de floresta ribeirinha na bacia do rio Piratini, Rio Grande do Sul, Brasil. Biotemas, Florianópolis, v. 22, n. 3, p. 47 - 55, 2009.

SOARES, A. P.; SOARES, P. C.; HOLZ, M. Correlações estratigráficas conflitantes no limite PermoTriássico no Sul da Bacia do Paraná: o contato entre duas sequências e implicações na configuração espacial do aquífero Guarani. Revista Pesquisas em Geociências, v. 35, n. 2, p. 115 - 133, 2008.

SOBRAL, M.; JARENKOW, J. A. (Orgs.). Flora arbórea e arborescente do Rio Grande do Sul, Brasil. São Carlos: Rima; Porto Alegre: Novo Ambiente, 2006. 350 p.

SÜHS, R. B.; PUTZKE, J.; BUDKE, J. C. Relações florístico-geográficas na estrutura de uma floresta na região central do Rio Grande do Sul, Brasil. Floresta, Curitiba, v. 40, n. 3, p. 635 - 646, 2010.

VACCARO, S.; LONGHI, S. J. Análise fitossociológica de algumas áreas remanescentes da Floresta do Alto Uruguai, entre os rios Ijuí e Turvo, no Rio Grande do Sul. Ciência Florestal, Santa Maria, v. 5, n. 1, p. 33 - 53, 1995.

VAN DER PIJL, L. Principles of dispersal in higher plants. Berlin: Springer-Verlarg, 1982.

ZAR, J. H. Biostatistical analysis. New Jersey: Prentice Hall, 5.ed., 2009. 944 p. 
FLORESTA, Curitiba, PR, v. 43, n. 2, p. 241 - 254, abr. / jun. 2013. Scipioni, M. C.; Galvão, F.; Longhi' S. J. 